\title{
The design stance and its artefacts
}

\author{
Pieter E. Vermaas • Massimiliano Carrara • \\ Stefano Borgo - Pawel Garbacz
}

Received: 17 May 2010 / Accepted: 21 January 2011 / Published online: 12 February 2011

(C) The Author(s) 2011. This article is published with open access at Springerlink.com

\begin{abstract}
In this paper we disambiguate the design stance as proposed by Daniel C. Dennett, focusing on its application to technical artefacts. Analysing Dennett's work and developing his approach towards interpreting entities, we show that there are two ways of spelling out the design stance, one that presuppose also adopting Dennett's intentional stance for describing a designing agent, and a second that does not. We argue against taking one of these ways as giving the correct formulation of the design stance in Dennett's approach, but propose to replace Dennett's original design stance by two design stances: an intentional designer stance that incorporates the intentional stance, and a teleological design stance that does not. Our arguments focus on descriptions of technical artefacts: drawing on research in engineering, cognitive psychology and archaeology we show that both design stances are used for describing technical artefacts. A first consequence of this disambiguation is that a design stance,
\end{abstract}

\footnotetext{
P. E. Vermaas $(\varangle)$

Philosophy Department, Delft University of Technology, Jaffalaan 5, 2628 BX Delft, The Netherlands e-mail: p.e.vermaas@tudelft.nl
}

\section{Carrara}

Department of Philosophy, University of Padua, Padua, Italy

e-mail: massimiliano.carrara@unipd.it

\section{S. Borgo}

Laboratory for Applied Ontology, ISTC-CNR, Trento, Italy

e-mail: borgo@loa-cnr.it

S. Borgo

KRDB, Free University of Bolzano, Bolzano, Italy

P. Garbacz

Department of Philosophy, John Paul II Catholic University of Lublin, Lublin, Poland e-mail: garbacz@kul.lublin.pl 
in terms of interpretative assumptions and in terms of the pragmatic considerations for adopting it, stops to be a stance that comes hierarchically between the physical stance and the intentional stance. A second consequence is that a new distinction can be made between types of entities in Dennett's approach. We call entities to which the intentional designer stance is applied tools and entities to which the teleological design stance is applied instruments, leading to a differentiated understanding of, in particular, technical artefacts.

Keywords Design stance - Technical artefact - Daniel C. Dennett · Engineering · Cognitive psychology $\cdot$ Archaeology $\cdot$ Classes of artefacts $\cdot$ Instrument $\cdot$ Tool

\section{Introduction}

Technical artefacts slowly make their way towards a more centre position in philosophy. They have surfaced occasionally in the limelight, say in the work of Francis Bacon, Julian de la Mettrie and Martin Heidegger, but received more systematic analysis only within the specialist niches of philosophy of technology and science and technology studies. The emerging interest is partly motivated by more traditional philosophical projects, such as analyses of the mind, biological organisms, and art and fiction. Yet artefacts are increasingly also considered in their own right, leading to a fusion of research originating from distant fields such as philosophy of technology, science and technology studies, engineering design, cognitive psychology and metaphysics. ${ }^{1}$ This emerging interest therefore poses an interesting challenge to philosophy for collaborating across traditional disciplinary divisions, and has the promise to advance our understanding of technical artefacts, to the benefit of all disciplines involved.

In this paper we take up the sketched promise by revisiting the work by Daniel C. Dennett on the design stance with the aim of developing it and of showing that the type of entities that is in philosophy typically lumped together under the general label of technical artefact may be analysed as consisting of different types. Specifically we consider Dennett's design stance as defined and described by examples in his (1978, 1987, 1990, 1995). We show that there are two ways of spelling out this design stance, a teleological way that does not presuppose also adopting Dennett's intentional stance, and a second intentional way that does include the intentional stance. We argue against taking one of these ways as the correct formulation of the design stance in Dennett's approach, but propose to disambiguate Dennett's original design stance and to replace it by a teleological design stance that does not incorporate the intentional stance, and an intentional designer stance that does. Our argument focuses on the description of technical artefacts. Drawing on research in engineering, cognitive psychology and archaeology we show that both design stances are used for describing technical artefacts, establishing that each is needed in Dennett's approach towards interpreting entities.

\footnotetext{
1 Recent work on artefacts can be found in volumes by Costall and Dreier (2006) and Margolis and Laurence (2007), in special issues by Kroes and Meijers (2002, 2006) and Houkes and Vermaas (2009a), and in monographs by Baird (2004), Elder (2004), Lewens (2004), Verbeek (2005), Baker (2007), Thomasson (2007) and Houkes and Vermaas (2010). Earlier work includes Hilpinen (1992, 1993) and Dipert (1993).
} 
We argue that by the disambiguation of the design stance the relations between the different stances in Dennett's approach can be clarified. In Dennett's original formulation it is unclear whether the design stance makes weaker or stronger interpretative assumption than the intentional stance. When disambiguated, it can be observed that the teleological design stance makes weaker assumptions than the intentional stance, and that the intentional designer stance makes stronger assumptions. In Dennett's original formulations the pragmatic considerations to replace one stance by another in describing an entity seems linear: one switches from the physical stance to the (original) design stance because the latter may be more efficient in predicting the behaviour of an entity, and one switches from this design stance to the intentional design stance because the latter may be even more efficient. Conversely, when predictions by the intentional stance are inaccurate, one falls back on the design stance for describing an entity, and when the design-stance predictions are in accurate, one falls back to the physical stance. When disambiguated, the pragmatic ordering of the stances becomes more complex. Finally we use the disambiguation to draw a distinction between two types of entities: entities to which the teleological design stance is applied, which we call instruments, and entities to which the intentional designer stance is applied, which we call tools. The proposed disambiguation thus leads to a more differentiated understanding of entities in Dennett's approach. The distinction between instruments and tools can moreover be related to a more liberal and stricter approach in philosophy towards demarcating technical artefacts.

In Sect. 2 we introduce Dennett's three stances and in Sect. 3 we describe the ambiguity in Dennett's writing. In Sect. 4 we consider existing conceptual analyses and criticisms of the design stance in philosophy. In Sects. 5 and 6 we review empirical work on artefacts in engineering design methodology, cognitive psychology and archaeology for showing that technical artefacts are described by both the teleological design stance and the intentional designer stance. Then, extending Dennett's approach, we introduce in Sect. 7 the teleological design stance and the intentional designer stance in more detail and discuss the ordering of the stances by their interpretative assumptions and by the pragmatic considerations for adopting them. In Sect. 8 we introduce the distinction between instruments and tools.

\section{Dennett's three stances}

Consider any entity whose behaviour you are interested in to predict. Such an entity could be represented as a simple object, as for example a rock or a spoon, or as a very complex entity, as a suricat, a smartphone or a human being. In Dennett's view we use three stances to describe these entities for predicting their behaviour: a physical stance, a design stance and an intentional stance.

When we adopt the physical stance towards an entity "our predictions are based on the actual physical state of the particular [entity], and are worked out by applying whatever knowledge we have of the laws of nature" (Dennett 1978, p. 4).

The second stance is the design stance. With this stance we predict the behaviour of an entity by appeal to the assumption that it is a designed entity. For Dennett this assumption of design means that the entity can be broken up in parts that have specific 
functions, where function is a "purpose-relative or teleological" notion. Predictions by means of the design stance are then generated by the assumptions that the parts are optimally suited for performing the functions assigned to them, and that these functional parts are not malfunctioning. Paraphrasing Dennett, if one knows exactly the functions of each part of a radio, one can give a design-stance prediction of how the radio behaves when assuming that each part functions properly (1978, p. 4).

The third stance is the intentional one. It is a stance where we predict what an entity will do by appeal to the assumption that it is a rational agent with certain overarching goals and certain perceptual and behavioural capacities. Adopting the intentional stance you first

decide to treat the [entity] whose behavior is to be predicted as a rational agent; then you figure out what beliefs that agent ought to have, given its place in the world and its purpose. Then you figure out what desires it ought to have, on the same considerations, and finally you predict that this rational agent will act to further its goals in the light of its beliefs. A little practical reasoning from the chosen set of beliefs and desires will in many-but not all-instances yield a decision about what the agent ought to do; that is what you predict the agent will do. (Dennett 1987, p. 17, original emphasis)

The physical stance seems to be the one that can always be adopted for predicting the behaviour of an entity. But it is also the most cumbersome one. For the rock or spoon it may be practically feasible but for entities as radio sets, suricats, smartphones or human beings, application of the physical stance becomes quickly too complex. For these latter entities it is more efficient to adopt the design stance or the intentional stance. The choice which of the three stances to adopt for predicting the behaviour of an entity is thus primarily a pragmatic choice, and not one based on a pre-established position that the entity involved is actually a designed entity with functional parts or an entity with beliefs on which it acts rationally.

Since for Dennett the choice to adopt one of the stances does not presuppose a pre-established position about the actual nature of the entity, the design stance and the intentional stance can be applied to all sorts of entities. The design stance can be applied to radio sets but also to genes and biological entities in general, and the intentional stance can be applied to persons but also to chess-playing computers, animals and Martians. A successful application of the design stance or of the intentional stance to an entity-where success means that the generated predictions are accurate-shows for Dennett that the entity can be taken as, respectively, a designed entity or an agent (in Dennett's sense). Moreover, the choice to adopt the design stance also does not presuppose a pre-established position about the specific purpose and functions of the entity concerned. And the choice to adopt the intentional stance does, similarly, not presuppose a pre-established position about the specific goals and beliefs of the agent. These purposes and functions, or goals and beliefs, are rather derived: a successful application of the relevant stance to the entity advances specific purposes and functions, or goals and beliefs (Vaesen and Van Amerongen 2008, p. 783). Taking genes as molecules with specific interests (Dennett 1995, p. 328) and taking biological entities generally as designed, as Dennett systematically does, is thus not due to a 


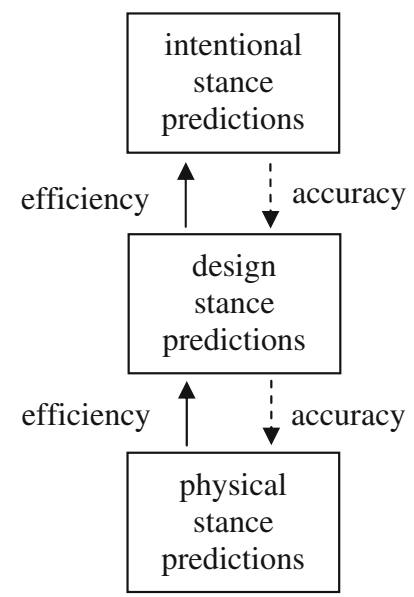

Fig. 1 Pragmatic ordering of Dennett's original three stances

pre-established position about these entities having interests or functions, but due to the successes of predicting their behaviour with the design stance.

\section{The ordering of the stances}

Dennett sometimes introduces his three stances in the order as we presented them above (e.g., 1987, pp. 16-17) and this ordering also seems to be reflected by the pragmatic considerations that govern their adoption. The physical stance can always be used for predicting the behaviour of entities, but for some entities it is more efficient to arrive at these predictions by applying the functional descriptions of the design stance. And for some of those entities the efficiency of the design stance is in turn outclassed by the intentional stance. Reversely, if for an entity for which one has chosen the intentional stance one observes that the entity does not behave rationally, then one abandons the intentional stance and can decide that adopting the design stance is more effective in predicting its behaviour. And if an entity for which we have adopted the design stance is observed to have parts that are malfunctioning, we switch to the physical stance (Dennett 1978). In Fig. 1 the ordering of the three original stances by means of these pragmatic considerations is depicted schematically, where a normal arrow represents the consideration to switch from one stance that produces accurate predictions about an entity to another stance for acquiring more efficient predictions, and where a dotted arrow represents the consideration to switch from one stance that does not produce accurate predictions about an entity to another stance that does generate such accurate predictions.

This ordering would also reflect the strength of the assumptions made in the stances. When adopting the physical stance, one assumes uncontroversially that the behaviour of entities can be described by their physical structure and the laws of physics. When adopting the design stance, one adds a teleological perspective and ascribes purposes 
to entities. And when adopting the intentional stance, one assumes that the entities not only have goals but are also agents with beliefs on which they act rationally.

A more detailed look shows however a more complicated relationship between specifically the design stance and the intentional stance. Dennett argues at length that when we determine the functions and purposes of artefacts, the intentions of the designers are not relevant (e.g., Dennett 1990, pp. 184-186). He discusses cases in which such intentions are indeed irrelevant, as when one buys an old-fashioned sadiron for using it as a doorstop rather than for ironing, or when one anchors a boat with a large mainframe computer (1990, p. 184). Yet, in a number of the analyses and examples that Dennett gives of the design stance, elements of the intentional stance are mentioned as well. When he describes the design stance adopted for the chess-playing computer, Dennett talks about deriving its behaviour on the basis of knowledge of how the computer is designed $(1978$, p. 4). In principle one can understand this knowledge as knowledge about the purposes and functions that the person who is applying the design stance to the computer is imposing on the computer. But it is more faithful to Dennett's writing to understand this knowledge as knowledge of how the chess-playing computer was intentionally designed by the software developers. This second way of understanding introduces agents within the design stance, that is, agents to whom, as part of applying the design stance to the computer, the intentional stance is applied. Moreover, the analyses and examples in which Dennett refers to designing agents are not limited to those concerning technical artefacts. When considering adopting the design stance for biological entities, Dennett talks frequently about 'Mother Nature' who is doing the designing. Again, in principle there is no need to introduce this additional entity; when adopting the design stance for biological entities, it suffices to just assume that the biological entities have purposes and functions, and then derive these purposes and functions as part of a successful prediction of their behaviour. Yet Dennett typically does introduce Mother Nature. According to him we apply the intentional stance to natural selection and then discover how she-Mother Nature-has designed biological entities (1990, p. 187). In his (1995) Dennett seems to include his intentional stance more explicitly into the design stance when he explains the interpretation of biological entities as if they were technical artefacts. Dennett does this by means of the engineering technique of reverse engineering, and describes reverse engineering of a technical artefact in turn as a reconstruction of the reasons the original engineers had when designing the artefact (pp. 212-213). Hence, according to Dennett's writing, when adopting the design stance for describing an entity, one puts a teleological-functional perspective on the entity and assumes in a number of central cases - chess-playing computers, biological entities - the presence of an agent who designed that entity. This observation supports an understanding of the design stance as one in which the intentional stance is included, and would, in terms of interpretative assumptions, put the design stance third in row, since then application of the design stance implies the assumption that the entity concerned has a purpose and functional parts, and the assumptions that come with the intentional stance that there is a designer of the entity who has goals and beliefs, and acts rationally on the basis thereof.

One way to resolve this issue how to order the stances is to argue in favour of one particular understanding of Dennett's design stance, be it the weaker one in which application of the design stance means that it is only assumed that an entity has parts 
with functions relative to some purpose, or be it the stronger one in which application of the design stance means also assuming that the entity has been designed by an agent for these functions and purposes. A second way to resolve the issue is to take the confusion about how to understand Dennett's design stance as an indication that his approach actually involves two design stances, which should both be articulated and accepted as separate design stances. In this paper we argue for this second resolution, and for this we focus in Sects. 5-6 on the description of technical artefacts. We show that for technical artefacts both design stances are in use, demonstrating that rejecting one of them would limit the domain of application of Dennett's approach towards describing entities.

Let us now reserve the name 'teleological design stance' for the weaker version of the design stance, and introduce the name 'intentional designer stance' for the stronger intentional version. By accepting both within Dennett's approach the ordering of the stances with respect to the strength of the interpretative assumptions made, becomes again clear: the physical stance comes first since it does not involve additional assumptions; the teleological design stance second because it adds the teleological perspective of purposes and functions to the description of entities; the intentional stance is third with the assumption that entities are also agents with beliefs on which they act rationally; and finally one has the intentional designer stance where one adopts the teleological perspective and the assumption that this perspective is due to a rational agent who has goals and beliefs and acts rationally. In Sect. 7 we continue discussing the two design stances and return to the ordering of the resulting four stances, in part because the pragmatic considerations for adopting one of the stances now becomes more complicated, and less linear as depicted in Fig. 1. But first we turn to philosophy, in which Dennett has been criticised under the presupposition that there is only one correct formulation of the design stance.

\section{Philosophy}

In philosophical analyses of Dennett's accounts three main positions on the relation between the design stance and the other stances have been developed:

- Eliminativism: the design stance is eliminable or reducible either to the physical stance or to the intentional stance.

- Primitivism: the design stance is irreducible and is assumed within the intentional stance.

- Intentionalism: the design stance presupposes the intentional stance applied to a designer.

Baker (1987) argued for eliminativism starting from the ontological observation that some features of entities are independent of one's predictive strategies, say the feature of water to freeze at $0^{\circ} \mathrm{C}$, and that other features are not, say the feature that someone has when believing that water freezes at $0^{\circ} \mathrm{C}$. Baker argues that this distinction can be motivated by Dennett's ontology (pp. 152-153) and then uses it to criticise Dennett. Rationality, for instance, is in Dennett's approach a feature that is attributed to agents by the intentional stance. So, rationality is a stance-dependent feature of agents, yet "Dennett often implies that it is a stance-independent feature, a feature that 
an organism has per se, without regard to the predictive strategies of others." (1987, p. 159)

In the case of the design stance Baker focuses in her arguments on design features of entities, being those features that warrant the application of the design stance to these entities. One of these features is that the entity concerned does not malfunction (or has been broken down). For artefacts the concept of malfunction is according to Baker a stance-dependent feature of the artefacts: it is dependent on the intentional stance since it refers to the intentions of the designers or users of the artefacts. This stance-dependence of malfunction now creates a dilemma in Dennett's approach when considering organisms to which the design stance is applied. If for organisms, malfunction is also a feature that is dependent on the intentional stance, then the features that warrant the application of the design stance to organisms (and artefacts) depend on the intentional stance, which counters Dennett's view that designs of organisms evolve by natural selection, and which makes that "the design level tends to collapse into the intentional." (1987, p. 161) If, however, malfunction is a stance-independent feature of organisms, a uniform treatment of organisms and artefacts is lost, and restoring that uniformity by taking design features such as malfunction stance-independent for both organisms and artefacts makes that "the design level tends to collapse into the physical" (p. 161).

The second position on the design stance, primitivism, has been put forward by Millikan (2000). Millikan starts by spelling out what she takes as the design stance, after noting an ambiguity in Dennett's proposal. This ambiguity is the following one: when applying the design stance to an entity, one can proceed from a description of the actual dispositions of the entity, or from a description of the dispositions it is supposed to have by its design, where design may refer to an intentional design as produced by designers or to natural design as produced by natural selection. Millikan opts for the second way: "[t]he design stance is the predictive stance that moves from what [an entity] was designed to do to a prediction that it will do that thing" (p. 57; original emphasis). Application of the design stance to an entity refers in this way to the origin of the entity in designing. Yet, a similar reference is absent in the way in which Dennett uses the intentional stance. The intentional stance can be applied to an entity on the basis of its actual dispositions only: if the entity exhibits behaviour that can be interpreted as rational behaviour, then it may be taken as an entity exhibiting rational behaviour, independently of what internal mechanisms are involved in this behaviour.

For Millikan this way of ascribing rationality to entities is too liberal and not providing support for the projectability of rationality, say from current behaviour dispositions to future dispositions. "[I]f some actual organism had acquired fully rational dispositions to behavior totally by accident, and if the observed appearances of rationality resulting from these dispositions were known by us to have resulted from accident, we would be foolish indeed to project these appearances into the future" (2000, p. 61). Something more is needed and for Millikan it is the design stance: "from design for rationality, one can infer real dispositions to rationality patterns, as opposed to mere temporary illusions of such dispositions. It thus appears that the intentional stance must be underwritten by the design stance, rather than vice versa" (pp. 61-62). 
The last position of intentionalism has been advanced by Ratcliffe (2001). For Ratcliffe the intentional stance is more fundamental than the design stance: "the intentional stance is not simply causally or epistemologically prior to the design stance but is also conceptually prior. Dennett's 'design stance' view of nature is simply unthinkable without a preconditional intentional stance" (p. 35).

Ratcliffe observes that a stance is a device that makes possible an interpretation, for example, an interpretation of the natural world as designed. For Dennett this interpretation is quite simply biology. But Ratcliffe observes: "[b]iology needs the concept of function and adaptation, and you get them from a construal of Mother Nature as a designer. Given this design construal, you can then understand intentionality in terms of biological function or design" (pp. 35-36). If this is so, design is only perceptible to one who employs the intentional stance. Then "the design stance is in fact ultimately derived from the intentional stance as opposed to the latter being a 'subspecies' of the former" (p. 36).

In general, for Ratcliffe, if we apply the design stance to an entity, then we should apply the intentional stance with respect to the designer of that entity. Ratcliffe takes this position from Fodor who argues that: "[d] esign (as opposed to mere order) requires a designer. Not theologically [..] but just conceptually. You can't explain intentionality by appealing to the notion of design because the notion of design presupposes intentionality" (Fodor 1996, p. 252; original emphasis).

Although it is not our aim to defend Dennett's approach to his critics, the disambiguation of Dennett's design stance in terms of the teleological design stance and the intentional designer stance as defined in the previous section, may provide elements to find a way out of some of the charges made. Disambiguation seems not to provide the means to defuse Baker's dilemma and her plea for eliminativism. The teleological design stance and the intentional designer stance are both meant to apply to technical artefacts and biological items uniformly, so for each of these stances, Baker can set up her dilemma. But take, secondly, Millikan's primitivism. The design stance that Millikan considers in her argument may on our disambiguation be taken as the teleological design stance as applied to organisms that display rational behaviour. Her conclusion can then be accepted, as Dennett (2000, p. 342) does, and be interpreted as revealing merely that the intentional stance presupposes the teleological design stance, not that the intentional stance presupposes both the teleological design stance and the intentional designer stance. This interpretation is coherent with the ordering of the stances as we argue for in Sect. 7. Consider, finally, Ratcliffe's arguments for intentionalism. From our perspective his arguments are showing the obvious conclusion that assumptions about the intentions of designing agents are part of the intentional designer stance. Yet, for the teleological design stance this conclusion need not be drawn.

\section{Engineering}

When one considers how in engineering technical artefacts are described, it seems that one is bound to accept intentionalism as defended by Ratcliffe. When in engineering a technical artefact is taken as designed, this straightforwardly means that it 
is assumed that the artefact has been intentionally designed by an agent. Moreover, when engineering functions are ascribed to parts of technical artefacts, these ascriptions presuppose designing agents, as we argue in this section. Hence, when spelling out Dennett's design stance, then engineering practices point towards the position that it should be unambiguously understood as what we have called the intentional designer stance. Yet, even in engineering one cannot suffice with only this intentional designer stance; some engineering descriptions involving functions are more accurately understood as descriptions that do not refer to designing agents. Hence, Dennett's design stance is better disambiguated and replaced by both the teleological design stance and the intentional designer stance.

Functions are used ubiquitously in engineering: technical entities such as devices and processes are in general described in structural and functional terms. Functions play specifically a pivotal role in methodologies for designing, redesigning and reverse engineering, and in descriptions of product architecture and engineering knowledge bases. Yet, despite its pivotal role, consensus on what is meant by function has not yet been achieved in engineering: a recent survey, for instance, listed 18 different meanings (Erden et al. 2008). In Borgo et al. (2009, 2010) we have given formalised definitions, grounded in a foundational ontology for information systems, of two of the main engineering meanings of function. These two meanings (identified in Chandrasekaran 2005) are the Functional Representation meaning of Chandrasekaran and Josephson (2000) and the Functional Modelling meaning exemplified by Stone and Wood (2000). The formalisation of especially the Functional Representation meaning revealed references to designing agents, which can be made explicit already in the informal description of this meaning.

Chandrasekaran and Josephson (2000) actually consider a range of engineering meanings of the concept of function, which they analyse as mixtures of two extremes: a device-centric meaning of function, which is a desired effect by a behaviour of an entity described in terms of that behaviour, and an environment-centric meaning, which is that desired effect described in terms of elements external to the entity. Chandrasekaran and Josephson take the concept of behaviour of an entity as neutral with respect to the intentions of agents but readily acknowledge that also this term is dependent on the agent who models the entity by focussing on some behaviour and ignoring other ( $\mathrm{p}$. 170). Hence, by taking functions as desired effects of behaviour of entities, functions refer in two ways to intentions of agents: to those of the modeller of the entity and to those who desire the effects. An example Chandrasekaran and Josephson use is that of an electrical buzzer: its device-centric function is making sound and its environmentcentric function is to enable a visitor to inform a person in a house that someone is at the door. These functions as effects are desired by the users and by the engineers who designed the buzzer, and these functions refer to a modelling of the behaviour of the buzzer as selected by the designing engineers.

Stone and Wood (2000) take a function of an entity as a relationship between the input and output flows of energy, material and signals of the entity, where this relationship has the purpose of performing an overall task as set by customers (p. 359). By this reference to the task of the entity, functions of entities can be reconstructed as intentionally determined by designers to meet the tasks as set by customers. An example is now a popcorn popper, which has the customer task to pop corn, and whose overall 
function is determined by designers as transforming a flow of corn kernels, electricity, butter and an on-off signal, to a flow of popcorn, thermal energy and acoustic energy.

Assuming that the discussed meanings of function are representative of engineering, it follows that taking an entity as having parts with functions implies in engineering that one is referring to intentions of designing agents. The conclusion is then that in engineering, Dennett's design stance presupposes the intentional stance. This conclusion may be avoided by denying that the functions in Dennett's design stance are the functions as considered in engineering, but such a strategy is in contradiction with his references to (reverse) engineering when Dennett explains his design stance. In engineering Dennett's design stance is thus to be spelled out as the intentional designer stance, that is, as a design stance that refers to intentionally designing agents. Yet, this conclusion cannot always be drawn in engineering. As announced in the beginning of this section, there are cases in which functional descriptions of entities in engineering do not refer to designing agents. Such cases include engineering explanations of why entities display or have displayed specific behaviour, such as after an accident of some sort. When an explosion takes place in a gas tank, an engineering investigation may reveal that an electrical switch, by giving a spark, functioned as a detonator. And when it is not a criminal investigation or a case of reverse engineering, it is not part of the explanation that an agent designed the switch for having the engineering function to detonate the gas. Such explanations can therefore be taken as ones in which the teleological design stance is applied. Given that engineering functions are referring to designer intentions, it has however now to be defended that in functional descriptions in the sketched explanations, engineers do not assign engineering functions to the parts of those entities. That this defence is possible can be made plausible by arguing that in, say, the explanation of the gas explosion engineers conclude that the switch functioned as a detonator but does not have detonating as its proper engineering function. We will not give this defence, but define in Sect. 7 what type of functions engineers assign in such explanations. Pending this definition, the conclusion is that in engineering descriptions both the intentional designer stance and the teleological design stance is applied, providing support to disambiguate Dennett's design stance in terms of these two more precise design stances, rather than taking one of them as the only correct one.

\section{Cognitive psychology and archaeology}

Some of Dennett's examples of how we describe technical artefacts already show that in some cases we do determine the functions and purposes of artefacts without referring to the intentions of designers. Examples like the mentioned sad-iron used as a doorstop are sufficient proof that the behaviour of some technical artefacts can be predicted on the basis of merely the assumptions that they have purposes and their parts functions, where the functions are optimal for letting the artefacts realise their purposes. Hence we indeed can apply the teleological design stance to technical artefacts. This claim does however not hold in general, as recently argued by Vaesen and Van Amerongen (2008) using the results of research in cognitive psychology and practices in archae- 
ology. They considered two ways of understanding Dennett's claims: as descriptive claims that persons, in artefact hermeneutics, reason in terms of optimality, rather than in terms of intentions; and as normative claims that persons, in artefact hermeneutics, should reason in terms of optimality, rather than in terms of intentions (2008, p. 782). Vaesen and Van Amerongen argue that both these ways are untenable.

For challenging the descriptive way of understanding Dennett's claims, Vaesen and Van Amerongen turn to cognitive psychology, in which they discern an essentialist tradition in describing how persons infer what artefacts are for, and an anti-essentialist tradition. Their conclusion is that within both traditions "it is safe to say that, according to cognitive psychology, designer's intentions play an important role in the interpretation of artifacts - the main topic of disagreement [between proponents of the two traditions] being exactly how important" (pp. 786-787). In the various experiments described, ranging from artefacts made for one reason but used for another, or artefacts made for one reason but not quite suited for that job, the original intentions of the designers of artefacts were shown to be part of the determination of what the artefacts are for. According to the essentialist tradition persons have a cognitive capacity to categorise and reason about artefacts on the basis of the original intentions of the designer. And although persons may categorise artefacts differently than by original intent according to the anti-essentialist tradition, designer's intentions are still an important feature for the categorisation of artefacts in that second tradition, although "not the only one" (p. 786).

For assessing the normative way of understanding Dennett's claims, Vaesen and Van Amerongen consider archaeology, in which functions of artefacts are determined by methodological rules. It is shown that archaeologists indeed formulate rules to reasoning in terms of optimality when discovering the functions of artefacts. Yet, Vaesen and Van Amerongen also show that these rules have their limitations and may lead to controversial conclusions. These limitations are illustrated with the determination of the function of Babylonian vessels that may be used as galvanic cells and have therefore been called Babylonian batteries. On the basis of optimality considerations the conclusion may indeed be that these vessels have the function of providing electricity. This conclusion is however controversial, the alternative hypothesis being that the vessels were containers of sacred scrolls. This latter conclusion about the function of these vessels can however (only) be argued for when also the intentions of the Babylonians are taken into account. The use of the metals of iron and copper in the vessels, which makes them suitable as batteries, is then explained by referring to the beliefs of the creators of the vessels that these metals protected the scrolls against divine terror (2008, pp. 788-789). Hence, in archaeology functions of artefacts may be determined not only on the basis of ascribed functions and optimality considerations but also by taking into account the intentions of the agents who designed or constructed the artefacts.

These results again establish that Dennett's design stance should not be spelled out as either the teleological design stance or the intentional designer stance. Both design stances are in use when describing technical artefacts, supporting the position that Dennett's design stances should be disambiguated. 


\section{Four stances}

Let us take stock. In Sect. 3 we analysed the design stance as defined and described by Dennett. We argued that it is unclear whether or not this design stance should be understood as including assumptions about designing agents. Two responses were identified. One can argue in favour of one particular understanding of Dennett's design stance, or disambiguate and replace Dennett's design stance by two: a teleological design stance in which no references are made to designing agents; and an intentional designer stance in which such references are included. In Sect. 4 we reviewed philosophical analyses and criticisms of Dennett's design stance in which it is presupposed that there is only one way of spelling it out, and showed that disambiguation provides elements to resolve some of the criticisms. In Sects. 5 and 6 we argued for this response of disambiguation by showing that for technical artefacts both the teleological design stance and the intentional designer stance are in use. Hence, replacing Dennett's original design stance by these two design stances makes Dennett's approach empirically more faithful to how agents actually predict the behaviour of entities. Yet, for letting the disambiguation be conclusive we still have to fix a concept of function for the teleological design stance such that references to designing agents are indeed absent in that stance-functions may refer to designing agents, as argued in Sect. 5, and those references should be avoided for the teleological design stance. We carry out this task by adopting for both the teleological design stance and the intentional designer stance the concept of function as introduced by Searle (1995).

According to Searle (1995, pp. 13-23) functions are capacities that agents assign to parts of entities relative to values or purposes these agents impose on these entities (typically survival and reproduction for biological entities, and typically practical uses for artefacts). When this concept of function is adopted in the two new design stances, one has to spell out which agents are involved in the assignment of functions. This can be done in such a way that in the teleological design stance no assumptions are made about the intentions of other agents, whereas in the intentional designer stance such assumptions are made. Moreover, one can obtain an analysis of the functions assigned in the intentional designer stance that is by and large consistent with assuming that these functions are engineering functions. Let us start with the teleological design stance. The functions and purposes of entity $x$ and its parts can within this stance be taken as functions and purposes that are assigned relative to values or goals of the agent $y$ who applies the teleological design stance to $x$. Taking parts of entities as having functions then does not imply a reference to other agents; there are only references to the goals and values of the agent $y$ that applies the teleological design stance. When, for instance, the light switch that sparked the accidental gas explosion is functionally described as a detonator, it is not assumed that the switch has been designed by an agent for this function. Consider second the intentional designer stance. Now the functions and purposes of entities and their parts should not be taken as functions and goals that the agent $y$ who applies the intentional designer stance assigns relative to his or her own goals; rather $y$ assumes that these functions and purposes are the ones that other agents $z$ have assigned to these entities and parts relative to their values or goals. Hence, the functions and purposes of entities are now referring to other agents $z$ : the agent $y$ who applies the intentional designer stance to, say, a buzzer, assumes that its 
function of making sound is the function the designer $z$ of the buzzer has assigned to this buzzer.

So, having fixed the relevant concepts of function, Dennett's original design stance can indeed be replaced by the teleological design stance and the intentional designer stance. As a result Dennett's approach specifies four stances for predicting the behaviour of entities of any type. The physical and intentional stances are still the ones as defined and described by Dennett. In the teleological design stance a person $y$ predicts the behaviour of an entity $x$ by appeal to the assumption that $x$ is an entity with a purpose and with parts that have functions that are all assigned by person $y$. In the intentional designer stance a person $y$ predicts the behaviour of an entity $x$ by appeal to the assumption that $x$ is an entity with a purpose and with parts that have functions, and by appeal to the assumption that this purpose of $x$ and the functions are assigned by an entity $z$ that person $y$ describes as a rational agent with certain overarching goals and certain perceptual and behavioural capacities.

In terms of the strength of their assumptions the four stances again can be ordered linearly. When adopting the physical stance, one assumes uncontroversially that the behaviour of an entity $x$ can be described by its physical structure and the laws of physics. When adopting the teleological design stance, one adds a teleological perspective to the description of the entity. In the intentional stance the assumption is accepted that this entity $x$ is also an agent with beliefs on which it acts rationally. And the intentional designer stance involves the teleological perspective for $x$, the assumption that there is a second entity $z$, and the assumption that $z$ is a rational agent.

This linear ordering of the four stances is however not reflected anymore by the pragmatic considerations that govern their adoption. With the teleological design stance taking the place of Dennett's original design stance one has pragmatically still the linear ordering between the physical stance, the teleological design stance and the intentional stance, similar as the ordering depicted in Fig. 1 in Sect. 3. The physical stance can still always be used for predicting the behaviour of entities. For some entities these physical-stance predictions are laborious, and switching to the teleological design stance may lead to more efficient successful predictions. And for some entities the teleological-design-stance predictions may be outclassed in efficiency by the intentional stance. Conversely, if the predictions by the intentional design stance are not accurate, one falls back to the teleological design stance, and if these teleological-design-stance predictions are not accurate as well, one may switch back to the physical stance.

With the intentional designer stance added to the picture, new pragmatic changes become available relative to this linear ordering. When teleological-design-stance predictions of the behaviour of an entity are not accurate, one need not necessarily fall back to the physical stance; the intentional designer stance may be an alternative as well. Take the example of the Babylonian vessels. Application of the teleological design stance to these vessels leads by optimality considerations to the prediction that they are for generating electricity. Rejecting these predictions, more accurate ones are generated by the intentional designer stance. A question that now emerges is when one should switch back from the teleological design stance to the physical stance or when it makes sense to try the intentional designer stance. A first answer could be as follows. In the teleological design stance the purposes assigned to entities and the functions 
assigned to their parts are assigned by the predictor $y$ on the basis of optimality considerations about the entities and the parts. These optimality considerations are based on $y$ 's knowledge about what entities and their parts can do. If now the predictions generated by means of these assigned purposes and functions are not successful, and there are others purposes and functions available that, when assigned to entities, do lead to successful predictions, then it makes sense to switch to the intentional designer stance provided that these alternative purposes and functions can be assigned on the basis of optimality considerations based on the knowledge of the assumed designer $z$. If, in contrast, such other purposes and functions are not available, one falls back from the teleological design stance to the physical stance.

When one has successful intentional-designer-stance predictions for an entity, and teleological-design-stance predictions are as successful, the latter are more efficient: describing a radio set teleologically by means of purposes and functions an assumed designer $z$ would assign to the set is more laborious than describing the radio set by means of purposes and functions one assigns oneself. Starting from an intentional designer stance description of an entity $x$, a purely intentional description of that entity is more efficient as well. Consider the behaviour of cellular automata. Predicting their behaviour by means of their functional make-up and the intentions for which they were designed is possible but less efficient than predicting their behaviour by assuming they are intentional entities. Again the question emerges when one should try to replace an intentional-designer-stance description with one by the teleological design stance or one by the intentional stance. Complexity may be a criterion, where a more complex functional description of the entity can be taken as a reason to first try the intentional stance. When the predictions by means of the intentional designer stance are not accurate, then one can reconsider the beliefs and goals assigned to the assumed designer $z$, yet when that is not working, one ultimately has to fall back on the physical stance.

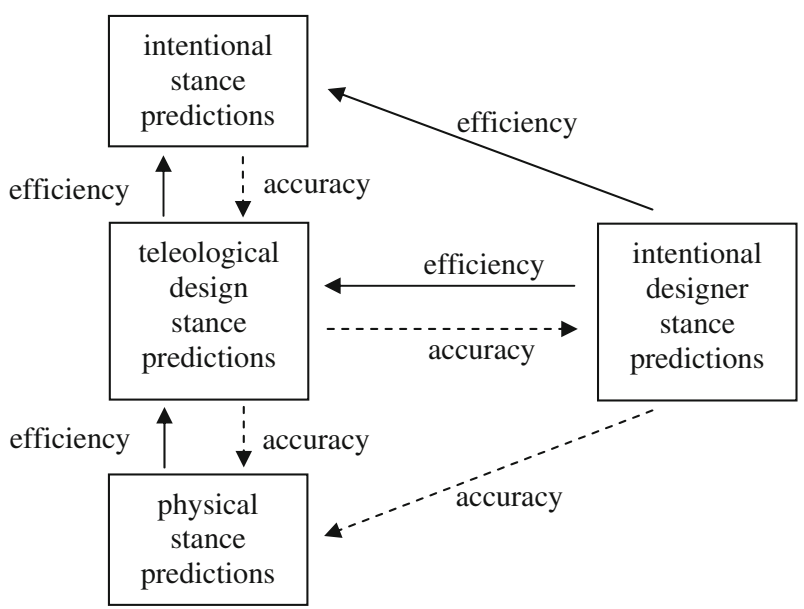

Fig. 2 Pragmatic ordering of the four stances 
The ordering of the four stances by means of the pragmatic considerations that govern their adoption is depicted in Fig. 2. The relations included are probably only the main ones. Given that one can have sequences in the switches between the stances, other relations may be included. One can, for instance, for an entity decide that a teleological-design-stance description is more efficient than a physical-stance description, and then conclude that an intentional-stance description is even better, arriving at an (indirect) "more efficient relation" between the physical stance and the intentional stance. More interesting is switching back from an inaccurate intentional-stance description to an intentional-designer-stance description. The behaviour of a software agent on internet may be irrational, accept if one assumes that the software has been designed by an agent with particular beliefs: for a European customer it is typically strange why software agents on Internet ask separately for the country in which one lives and the state in which one lives.

A final remark is that, as with all of Dennett's stances, one merely presupposes in the teleological design stance that $x$ has a purpose and its parts functions; one does not presuppose what these purposes and functions are. Also in the intentional designer stance the particular purpose and functions of $x$, and the particular goals and beliefs of $z$ are not assumed but determined and attuned such that the behaviour of $x$ is predicted successfully.

\section{Instruments and tools}

The proposed disambiguation of Dennett's design stance does not alter Dennett's overall project; the now four stances still define how we describe entities as diverse as rocks, suricats, smartphones and human beings, and they still give the means to identify how we understand such entities depending on what stance we adopt. If we adopt the physical stance for an entity to predict its behaviour, then according to Dennett we understand it as a physical entity. And if we adopt the intentional stance, we understand it as an agent. The disambiguation allows us to make a distinction between two types of what Dennett calls designed entities. If, in the original scheme, we adopt Dennett's original design stance for an entity, we understand it as a designed entity, where design may refer to intentional design or to design by other processes or mechanisms, such as natural selection. With the disambiguation, this understanding of entities as designed becomes more detailed and conceptually clearer. If we successfully and efficiently predict the behaviour of an entity with the intentional designer stance, then this entity is understood as an entity designed intentionally by agents. And if we adopt the teleological design stance for an entity, it is understood as an entity that has a functional structure through natural selection or simply by its complexity. Let us now call entities for which we adopt the teleological design stance instruments, and entities for which we adopt the intentional designer stance tools.

The proposed disambiguation also does not alter Dennett's position that we describe biological items and technical artefacts in the same way; the disambiguation merely details this position. The teleological design stance can be applied to biological organisms, to their organs and to their behaviour when a researcher assigns purposes and functions to the organisms on the basis of observation or of some theory such as 
evolutionary theory. And the teleological design stance can also be applied to technical artefacts and their components as in the case of explanations of accidents. Similarly the intentional designer stance can be applied to biological items by assuming that they are designed by Mother Nature, just as that this stance can be applied to technical artefacts. Disambiguation of Dennett's original design stance provides in this way the means to express the more daring elements of Dennett's position. Whereas the received view may be that biological items are physical entities or instruments because intentions have no role in biological explanation, Dennett holds that biological items are sometimes understood as tools as well, with Mother Nature as the designer. The detail that disambiguation brings can also be used for making more explicitly how we understand technical artefacts, an issue to which we now turn.

In philosophy there are two approaches towards understanding the concept of technical artefact, or artefact in general. Technical artefacts are regularly understood as entities that are made by agents like human beings, which implies that artefacts are entities that come into existence when they are intentionally physically modified by those agents (e.g., Dipert 1993; Houkes and Vermaas 2009b; Hilpinen 1992, 1993 on a strict interpretation). Prehistoric pieces of flint and modern submarines are on this first approach instances of technical artefacts. Entities that are naturally occurring in the sense of being the products of unintentional physical or biological processes are consequently not technical artefacts, supporting the generally shared intuition that natural objects are not technical artefacts, and vice versa. Natural entities like pebbles and fallen tree trunks are on the first approach not technical artefacts. Given this approach talking about made technical artefacts is redundant. Yet, with the second more liberal approach it does make sense to talk about made technical artefacts. On that approach intentionally modified entities are artefacts but so are entities that are merely intentionally selected by agents to serve practical purposes (e.g., Borgo and Vieu 2009; Hilpinen 1992, 1993 on a liberal interpretation). Pebbles that are used as paperweights, and tree trunks that fell over streams and are used for crossing those streams, are then technical artefacts as well. These pebbles and tree trunks are often chosen as a starting point for philosophical analyses of technical artefacts or of technology, and such analyses sometimes remain on that level; an approach that may be somewhat limited from an engineering or contemporary everyday point of view (Houkes and Vermaas 2009b). Dipert's (1993) analysis of artefacts provides a more sophisticated and encompassing conceptual framework, giving a characterisation in which artefacts are defined in a stricter manner and contrasted to other types of more liberal 'technical' entities. ${ }^{2}$ With our disambiguation we can now characterise the first stricter approach towards techni-

\footnotetext{
2 Dipert (1993) discerns three types of artificial entities. Instruments form the broadest type and are entities that have been "intentionally used in intentional activity." More precisely, an instrument is an entity "one of whose properties has been thought by someone to be means to an end and that has been intentionally employed in this capacity", where an object is thought as a means to an end if it is conceived to make a net positive causal contribution to an end (pp. 24-25). Tools form a subtype of instruments: they are instruments intentionally modified for contemplated use (p. 27). Artefacts form in turn a subtype of tools: artefacts are intentionally modified tools "whose modified properties were intended by the agent to be recognized by an agent at a later time as having been intentionally altered for that, or some other use" (pp. 29-30). In our definitions we adopt Dipert's distinction between instruments and tools in terms of intentional modification, but do not adopt his emphasis on communication in his definition of artefacts. In fact we discern two definitions of artefacts: as tools or as instruments and tools.
} 
cal artefacts as one in which only tools are taken as technical artefacts, and the second more liberal approach as one in which both instruments and tools are acknowledged as technical artefacts.

We end with three caveats. The first concerns the special case in which a designer predicts the behaviour of an entity $x$ that s/he has designed her/himself. When such predictions refer only to the purposes and functions as assigned by the designer, then these predictions count as generated by the teleological design stance, and the conclusion is that the entity $x$ is by the designer $y$ taken as an instrument. This conclusion is in conflict with the intuition that designed entities $x$ are technical artefacts on any approach towards understanding artefacts. This conflict is resolved when the predictions by the designer $y$ are referring not only to the purposes and functions as assigned by $y$, but also to beliefs and knowledge of $y$, say to intentional choices to include particular components, or to engineering rules and technical principles about how technical structures work. These predictions may then be taken as generated by the intentional designer stance, and the conclusion is that the entity $x$ is a tool and thus a technical artefact on any approach. For this two roles of agent y should be discerned: the predictions of the agent $y$ should be analysable as predictions generated by $y$ in the role of predictor on the basis of purposes and functions assigned to the entity by $y$ in the role of designer.

The second caveat concerns predictions in which the predictor $y$ refers to an agent $z$ who has merely intentionally selected the entity $x$ for a specific purpose. As these predictions do not include functions of $x$ as assigned by $z$, one is not dealing with an application of one of our design stances. Hence, it is neither an instrument nor a tool, and thus not a technical artefact on any of the two approaches to technical artefacts. This conclusion contradicts a view held by Hilpinen (1993, pp. 170-171), who introduced the example of driftwood that became art after being selected and put on exhibition by an artist. Hilpinen takes this driftwood as an artefact, yet on our analysis it is not a technical artefact on any approach. ${ }^{3}$

The third caveat is a variation of the second that warrants more attention. It consists of the case that the predictor $y$ assumes that an agent $z$ intentionally selected an entity $x$ for a specific purpose and that $z$ assigned functions to parts of $x$. Such predictions should strictly speaking be taken as intentional designer stance predictions, which turns the entity $x$ into one that is to be understood as a tool and which makes that $x$ is a technical artefact also on the stricter approach. Yet this entity $x$ is not one that is made in the sense of being intentionally physically modified by an agent - it is merely intentionally selected. A way to avoid this conclusion is by arguing that the agent $z$ cannot be taken as a designing agent, since designing an entity implies that it has to be made or assembled rather than merely selected. The advantage of this first response is that entities that can be taken as tools are always made entities, which is in line with the stricter approach that technical artefacts are always made entities. A disadvantage is that one is eventually forced to introduce a fifth 'intentional selector stance' for describing the considered case. A second response may consist of arguing that in this case one should not use the intentional designer stance for predicting the behaviour

3 When considering artistic artefacts, more liberal approaches are possible; artistic artefacts may even include entities that are not assigned purposes (Carroll 1999, p. 148). 
of the selected entity, but the teleological design stance. If that leads to the same predictions, then the teleological design stance is the more efficient one, implying that $x$ is to be taken as an instrument after all. For pebbles and fallen tree trunks this argument may work: predictions about a pebble to be used as paperweights generated by the intentional designer stance and the additional assumption that someone selected the pebble as a paper weight, are in general also generated by the teleological design stance.

\section{Conclusions}

In this paper we analysed in detail Daniel C. Dennett's design stance and we argued that there are two ways of spelling it out. The design stance can firstly be taken as one in which the behaviour of an entity is predicted by appeal to the assumption that the entity has a purpose and parts with functions that serve that purpose. Secondly it can be understood as a stance in which the behaviour of an entity is predicted by appeal to the assumption that the entity has a purpose and parts with functions that serve that purpose, where the purpose and functions originate from an agent that has designed the entity. We argued against taking one of these formulations as the only correct one, but propose to replace Dennett's original design stance by two design stances, reserving the name teleological design stance for the design stance in which no references are made to designing agents, and introducing intentional designer stance as a term that refers to a design stance which includes such references.

Our arguments for incorporating both these design stances in Dennett's approach were drawn from engineering, cognitive psychology and archaeology, and consisted in establishing that technical artefacts are describes with both the teleological design stance and the intentional designer stance.

We have analysed the ordering of the stances with respect to their interpretative assumptions and with respect to the pragmatic considerations that govern their adoption. With the disambiguation of the design stance, the pragmatic ordering of the stances stops to be a simple linear ordering.

Finally we have drawn a distinction between tools and instruments. Instruments are entities for which we adopt the teleological design stance, and are understood as entities with purposes and functions we assign in our descriptions of them. Tools are entities for which we adopt the intentional designer stance, and are understood as entities with purposes and functions we assume in our descriptions to be assigned by their designers. The distinction between tools and instruments can in turn be used to distinguish between a strict and more liberal approach towards understanding entities as technical artefacts.

Our analysis prompts further analysis. The focus in our arguments and discussions of the design stance in Dennett's approach has been on technical artefacts, and not on biological items. This focus has been deliberate: we aimed to contribute to the emerging interest in technical artefacts and to show that this interest has promise for also more traditional disciplines in philosophy. The distinction between tools and instruments proved to be of use for a more differentiated understanding of technical artefacts. An issue for further analysis is how our analysis and specifically the distinction between 
instruments and tools can lead to a more differentiated understanding in the biological domain. Given Dennett's insistence that we understand biological organisms as designed by Mother Nature, turning those organisms into tools, a question may be which biological items are understood as not designed, i.e., as instruments. Items that come to mind are chemical processes within biological organisms, which at most may be taken as selected by nature, or items that play a role in detrimental biological processes, such as deceases and decay, and which can be taken as designed only when Mother Nature may also be a diabolical agent. Yet, with these observations we have stepped outside our domain of expertise.

A second issue concerns the way in which we have realised the disambiguation of the design stance. We did so by replacing Dennett's original design stance by the teleological design and intentional designer stances. An alternative way to proceed is to understand Dennett's original design stance strictly as a stance in which no references to intentional agents are made - that is, to interpret Dennett's original design stance as the teleological design stance-and then introduce the intentional designer stance as a composite of the design stance and the intentional stance. The physical, design and intentional stances can then be seen as the basic stances by which we predict the behaviour of entities, whereas the intentional designer stance is one that combines two of these basic stances.

This alternative has the benefit that the number of basic stances is again three as implied by Dennett's original proposal, yet leads to the further project of considering other stances that are composites of the physical, design and intentional stances. One can, for instance, envisage a 'design-design' stance, in which one predicts the behaviour of an entity $x$ by assumption that this behaviour of $x$ is in some way related to the behaviour of a second entity $z$, where the design stance is applied to both $x$ and $z$. For instance, when predicting the behaviour of a spider's web or a beavers' dam, one can apply this design-design stance to both the web and the spider or to both the dam and the beavers. Moreover, one can envisage that within combinations of the design and intentional stances other relations between the entity $x$ and the agent $z$ are considered than the relation of designing. In the third caveat at the end of Sect. 8 we already considered the possibility that an agent $z$ merely selects an entity $x$ for a practical purpose; this case may be taken as an instance of an 'intentional-design' stance in which the relation of designing is replaced by that of selection. Entities to which such an 'intentional selector stance' applies may be called means. The spectrum of composite stances one ends up with in this way may make Dennett's stance approach even conceptually clearer and empirically faithful to how agents actually predict the behaviour of entities. In this paper we looked at descriptions of technical artefacts in engineering, cognitive psychology and archaeology, but other areas have to be considered as well, including developmental psychology, for instance, for incorporating the teleological stance that Kelemen (1999a,b,c) introduced for capturing how children describe entities.

The spectrum of composite stances may also be useful for introducing further types of entities in the technical domain. A design-design stance as applied to entities that, informally, are used by animals, may be used to characterise and distinguish animal instruments and animal tools. And by considering different relations between the entity $x$ and the agent $z$ in the intentional designer stance, more fine-grained 
distinctions within the realm of technical artefacts may become available. Intentional designing and intentional modifying may eventually be distinguished in terms of the actions, beliefs and knowledge involved. The notion of tool may then be split in, for instance, the subtypes of artisan tools (modified but not intentionally designed) and of technological tools (modified and intentionally designed). And when accepting an intentional selector stance, one can make a distinction between means that are described by purposes and functions, and that are described by purposes only. It is our conjecture that by developing the spectrum of stances more distinctions between types of technical artefacts and technical entities can be drawn within philosophy. Yet we readily acknowledge that this spectrum of composite stances makes the overall picture more diffuse relative to Dennett's small set of three stances for understanding entities, and our set of four.

Acknowledgments We are grateful for helpful comments by Nathan Crilly, Krist Vaesen and two reviewers on earlier drafts of this paper. This work has been developed in part within the Marie Curie EuJoint project (IRSES 247503). Research by Pieter Vermaas was supported by the Netherlands Organisation for Scientific Research (NWO).

Open Access This article is distributed under the terms of the Creative Commons Attribution License which permits any use, distribution, and reproduction in any medium, provided the original author(s) and the source are credited.

\section{References}

Baird, D. (2004). Thing knowledge. Berkeley, CA: University of California Press.

Baker, L. R. (1987). Saving belief: A critique of physicalism. Princeton, NJ: Princeton University Press.

Baker, L. R. (2007). The metaphysics of everyday life: An essay in practical realism. Cambridge: Cambridge University Press.

Borgo, S., Carrara, M., Garbacz, P., \& Vermaas, P. E. (2009). A formal ontological perspective on behaviors and functions of technical artefacts. Artificial Intelligence for Engineering Design, Analysis and Manufacturing, 23, 3-21.

Borgo, S., Carrara, M., Garbacz, P., \& Vermaas, P. E. (2010). A Formalization of Functions as Operations on Flows. Manuscript.

Borgo, S., \& Vieu, L. (2009). Artefacts in formal ontology. In A. W. M. Meijers (Ed.), Philosophy of technology and engineering sciences (pp. 273-307). Amsterdam: Elsevier.

Carroll, N. (1999). Philosophy of art. London: Routledge.

Chandrasekaran, B. (2005). Representing function. Artificial Intelligence for Engineering Design, Analysis and Manufacturing, 19, 65-74.

Chandrasekaran, B., \& Josephson, J. R. (2000). Function in device representation. Engineering With Computers, 16, 162-177.

Costall, A. \& Dreier, O. (Eds.). (2006). Doing things with things: The design and use of everyday objects. Aldershot: Ashgate.

Dennett, D. C. (1978). Brainstorms: Philosophical essays on mind and psychology. Montgomery, VT: Bradford Books.

Dennett, D. C. (1987). The intentional stance. Cambridge, MA: MIT Press.

Dennett, D. C. (1990). The interpretation of texts, people and other artefacts. Philosophy and Phenomenological Research, 50S, 177-194.

Dennett, D. C. (1995). Darwin's dangerous idea: Evolution and the meaning of life. New York, NY: Simon and Schuster.

Dennett, D. C. (2000). With a little help from my friends. In D. Ross, A. Brook, \& D. Thompson (Eds.), Dennett's philosophy: A comprehensive assessment (pp. 327-388). Cambridge, MA: MIT Press.

Dipert, R. R. (1993). Artefacts, art works, and agency. Philadelphia, PA: Temple University Press. 
Elder, C. C. (2004). Real natures and familiar objects. Cambridge, MA: MIT Press.

Erden, M. S., Komoto, H., Van Beek, T. J., D’Amelio, V., Echavarria, E., \& Tomiyama, T. (2008). A review of function modeling: Approaches and applications. Artificial Intelligence for Engineering Design, Analysis and Manufacturing, 22, 147-169.

Fodor, J. (1996). Deconstructing Dennett's Darwin. Mind and Language, 11, 246-262.

Hilpinen, R. (1992). Artefacts and works of art. Theoria, 58, 58-82.

Hilpinen, R. (1993). Authors and artefacts. Proceedings of the Aristotelian Society, 93, 155-178.

Houkes, W., \& Vermaas, P. E. (2009a). Artefacts in analytic metaphysics: Introduction. Technè, 13, 74-81.

Houkes, W., \& Vermaas, P. E. (2009b). Contemporary engineering and the metaphysics of artefacts: Beyond the artisan model. Monist, 92, 403-419.

Houkes, W., \& Vermaas, P. E. (2010). Technical functions: On the use and design of artefacts. Dordrecht: Springer.

Kelemen, D. (1999a). Beliefs about purpose: On the origins of teleological thought. In M. Corballis \& S.E.G. Lea (Eds.), The descent of mind: Psychological perspectives on human evolution (pp. 278-294). New York: Oxford University Press.

Kelemen, D. (1999b). The scope of teleological thinking in preschool children. Cognition, 70, 241-272.

Kelemen, D. (1999c). Why are rocks pointy? Children's preference for teleological explanations of the natural world. Developmental Psychology, 35, 1440-1452.

Kroes, P. A., \& Meijers, A. W. M. (2002). The dual nature of technical artefacts: Presentation of a new research program. Technè, 6(2), 4-8.

Kroes, P., \& Meijers, A. (2006). The dual nature of technical artefacts. Studies in History and Philosophy of Science, A 37, 1-4.

Lewens, T. (2004). Organisms and artefacts: Design in nature and elsewhere. Cambridge, MA: MIT Press.

Margolis, E. \& Laurence, S. (Eds.). (2007). Creations of the mind: Theories of artefacts and their representation. Oxford: Oxford University Press.

Millikan, R. G. (2000). Reading mother nature's mind. In D. Ross, A. Brook, \& D. Thompson (Eds.), Dennett's philosophy: A comprehensive assessment (pp. 55-75). Cambridge, MA: MIT Press.

Ratcliffe, M. (2001). A Kantian stance on the intentional stance. Biology and Philosophy, 16, $29-52$.

Searle, J. R. (1995). The construction of social reality. New York, NY: Free Press.

Stone, R., \& Wood, K. (2000). Development of a functional basis for design. Journal of Mechanical Design, 122, 359-370.

Thomasson, A. (2007). Ordinary objects. Oxford: Oxford University Press.

Vaesen, K., \& Van Amerongen, M. (2008). Optimality vs. intent: Limitations of Dennett's artefact hermeneutics. Philosophical Psychology, 21, 779-797.

Verbeek, P.-P. (2005). What things do: Philosophical reflections on technology, agency, and design. University Park, PA: Penn State University Press. 